\title{
Anaemia among clinically well under-fives attending a community health centre in Venda, Limpopo Province
}

\author{
James Heckman, Amidou Samie, Pascal Bessong, Mmboniseni Ntsieni, Hassan Hamandi, Matthew Kohler, Benjamin Milam, \\ Jessica Scriver, Rebecca Dillingham
}

\begin{abstract}
Background. Anaemia has been reported to affect $20-75 \%$ of children in South Africa. The range suggests the effects that geography, health, and socio-economic status can have on the observed prevalence of anaemia within a specific community. Our objective was to investigate the prevalence of anaemia in children aged under 5 presenting for well-child examinations at a community health centre in Thohoyandou, Limpopo Province.

Design. A cross-sectional observational study was carried out in June and July 2007. Caregivers participated in a brief interview where demographic, health and nutritional information was collected. A blood sample was collected from each child, and haemoglobin levels were assessed with a point-of-care haemoglobin testing system. Anaemia was defined as having a haemoglobin value $<2$ standard deviations below age-altitude adjusted normal values.
\end{abstract}

Results. Three-quarters (39/52 - 75\%) of children were anaemic. Girls were significantly more likely to be anaemic than boys ( $20 / 20$ v. $19 / 32$ respectively; $p=0.001)$. Anaemic children were significantly less likely to be underweight compared with their peers ( $32 / 38$ v. $5 / 12$ respectively; $p=0.007)$. There was no significant association between anaemia and infection with Helicobacter pylori $(p=0.729)$, intestinal helminths ( $p=1.000)$ or food insecurity ( $p=0.515)$. Conclusion. We found a striking prevalence of anaemia among clinically well children $<5$ years old in Thohoyandou, Limpopo Province. The rates of anaemia were higher than those found in previous studies conducted in similar settings in South Africa. Future work should focus on aetiologies and interventions.

S Afr Med J 2010; 100: 445-448
In developing countries and resource-poor settings around the world, anaemia affects $46-66 \%$ of children; $80 \%$ of all children in these countries will suffer some degree of anaemia before their 18th birthday. ${ }^{1-3}$ The effects of childhood anaemia have been investigated across many disciplines, and range in severity from mild and reversible developmental impairment ${ }^{3-6}$ to greater risks of infection ${ }^{7}$ to mortality rates as high as $12.5 \%{ }^{8}$

In 1996, after a nationwide survey, the South African Vitamin A Consultative Group (SAVACG) reported that 1 in 5 South African children were anaemic $(\mathrm{Hb}<11 \mathrm{~g} / \mathrm{dl}) .{ }^{9}$ Subsequent reports showed anaemia to be present in $37-65 \%$ of otherwise well children in rural KwaZulu-Natal, ${ }^{10,11} 73 \%$ of HIV-infected children in Cape Town, ${ }^{12}$ and $9-10 \%$ of children in 2 informal

School of Medicine, University of Virginia, USA

James Heckman, 4th-year medical student

Hassan Hamandi, 3rd-year medical student

Matthew Kohler, 3rd-year medical student

Benjamin Milam, 3rd-year medical student

Jessica Scriver, 3rd-year medical student

School of Medicine and Department of Public Health Sciences, and Division of Infectious Disease and International Health, Center for Global Health, University of Virginia, Charlottesville, USA

Rebecca Dillingham, MD, MPH

Department of Microbiology, University of Venda, Thohoyandou, Limpopo

Amidou Samie, BSc, MSc, PhD

Pascal Bessong, PhD

Department of Nursing, University of Venda, Thohoyandou

Mmboniseni Ntsieni, nursing student settlements near Bloemfontein. ${ }^{13}$ These figures suggest the effects that geography, health and socio-economic status can have on the observed prevalence of anaemia within a given community, and validate Faber's observation that 'the values determined by only one survey, although national, cannot be extrapolated to specific geographical areas'.

We present a pilot study investigating the point prevalence of anaemia among children visiting a community health centre in Thohoyandou, Limpopo Province. We aimed to study the prevalence of anaemia in children presenting for well-child examinations, and to investigate possible aetiologies including malnutrition, ${ }^{10}$ intestinal helminth infection, ${ }^{1}$ and Helicobacter pylori infection. ${ }^{14}$

We also present preliminary results of a follow-up study conducted between June and August 2008 investigating the prevalence of anaemia among mother-child pairs in Thohoyandou.

\section{Methods}

\section{Patient selection}

Participants were recruited from children $<60$ months old receiving routine care at Thohoyandou Health Centre (THC) in Thohoyandou between June and July 2007. Communication with subjects and their families was assisted by Tshivendaspeaking assistants. The study was introduced at the conclusion of routine care and, if the child's parent or caregiver was interested, they were given time to ask questions and voice concerns. Informed consent was obtained. Children were excluded if they were clinically ill or $>60$ months old. 


\section{Study design}

The first encounter took place immediately after consent was given by the caregiver. Caregivers provided demographic information and a focused medical history (Table I), were given a $50 \mathrm{ml}$ screw-top sample jar, and asked to collect and return a stool sample as soon as possible. At the second encounter, the stool sample was collected, a haemoglobin level was obtained from capillary blood using a HemoCue 201+ point-of-care haemoglobin testing system, and household food security was assessed using the Household Food Insecurity Access Scale (HFIAS) survey. ${ }^{15}$ Subjects and their families were reimbursed for travel expenses. Data were recorded on paper encounter forms and transferred to a password-protected electronic database.

When it became apparent that few subjects returned for their second encounter, several were contacted; most cited childcare or domestic responsibilities as obstacles to revisiting. Therefore the first encounter was amended to include haemoglobin testing, administration of the HFIAS survey and, if possible, stool collection; patients were also given travel reimbursement in advance. If stool collection were not possible during the first encounter, subjects and their caregivers were asked to return the sample at a future date. If samples were not returned within a week, caregivers were phoned and encouraged to return the sample. If the caregiver were unreachable or still

Table I. Specific variables evaluated and their relation to prevalence of anaemia

\begin{tabular}{|c|c|c|}
\hline Variable & Anaemic (\%) & $p$ \\
\hline \multicolumn{3}{|l|}{ Gender } \\
\hline Male & 19/32 (59.4) & \multirow{2}{*}{0.001} \\
\hline Female & $20 / 20(100)$ & \\
\hline \multicolumn{3}{|l|}{ Food security } \\
\hline Secure & $25 / 32(78.1)$ & \multirow{2}{*}{0.515} \\
\hline Severely insecure & $13 / 19(68.4)$ & \\
\hline \multicolumn{3}{|l|}{$\begin{array}{l}\text { Dewormed in } \\
\text { previous } 6 \text { months? }\end{array}$} \\
\hline Yes & $2 / 3(66.7)$ & \multirow{2}{*}{1.000} \\
\hline No & $37 / 49(75.5)$ & \\
\hline \multicolumn{3}{|c|}{$\begin{array}{l}\text { Vitamin supplements } \\
\text { in previous } 6 \text { months? }\end{array}$} \\
\hline Yes & $8 / 12(66.7)$ & \multirow{2}{*}{0.466} \\
\hline No & $31 / 40(77.5)$ & \\
\hline \multicolumn{3}{|l|}{ Breastfeeding? } \\
\hline Yes & $30 / 39(76.9)$ & \multirow[b]{2}{*}{0.714} \\
\hline No & $9 / 13(69.2)$ & \\
\hline \multicolumn{3}{|c|}{ Current hookworm infection } \\
\hline Positive & 1/1 (100) & \multirow{2}{*}{1.000} \\
\hline Negative & $38 / 51(74.5)$ & \\
\hline \multicolumn{3}{|c|}{ Current $H$. pylori infection } \\
\hline Positive & 10/14 (71.4) & \multirow[b]{2}{*}{0.729} \\
\hline Negative & $29 / 38(73.1)$ & \\
\hline \multicolumn{3}{|l|}{ Stunted } \\
\hline Yes & $22 / 31(71.0)$ & \multirow{2}{*}{0.497} \\
\hline No & $14 / 17(82.4)$ & \\
\hline \multicolumn{3}{|l|}{ Underweight } \\
\hline Yes & $5 / 12(41.7)$ & \multirow[b]{2}{*}{.007} \\
\hline No & $32 / 38(84.2)$ & \\
\hline \multicolumn{3}{|l|}{ Wasted } \\
\hline Yes & $3 / 3(100)$ & \multirow{2}{*}{0.560} \\
\hline No & $32 / 44(72.7)$ & \\
\hline
\end{tabular}

unable to return to the clinic, study personnel made home visits to collect the samples.

In June 2008, investigators conducted a follow-up study investigating the prevalence of anaemia among mother-child pairs. Pairs were recruited from two clinic sites: THC and Pfanani Clinic (PC), both in suburban Thohoyandou. Informed consent was obtained. Children were excluded if $<6$ months or $>60$ months old.

The study comprised one visit. Assisted by Tshivendaspeaking research assistants, the mothers were asked to answer a survey assessing age, gender, recent health, nutrition, and household factors pertinent to anaemia. Haemoglobin levels were tested as in the 2007 study.

\section{Laboratory methods}

Stool samples were stored in the clinic freezer immediately after collection, transported to the University of Venda (UNIVEN)'s Department of Microbiology laboratory, and stored at $-20^{\circ} \mathrm{C}$. Once a week, new samples were thawed and screened for presence of hookworm oocysts by light microscopy, using the oocyst concentration method. Results were validated after completion of the study by a random cross-check of 10 samples by an independent microscopist. DNA was extracted and purified from each sample, using the QIAGEN Stool DNA Purification Kit. Polymerase chain reaction (PCR) analysis was carried out on each DNA sample to determine the presence or absence of $H$. pylori infection. ${ }^{16}$

\section{Patient follow-up and treatment}

Caregivers were notified of their child's results by post in the form of typed letters in English and Tshivenda that detailed the child's haemoglobin level and hookworm status. Patients were instructed to return to THC for treatment if the child were anaemic and/or positive for hookworms. The costs of treatment were covered by the health centre as per local protocol.

\section{Clinical definitions}

Anaemia. Normal haemoglobin levels vary as a child ages from birth to 60 months, and in children who live at sea level compared with those at an altitude of $1500 \mathrm{~m}$, as in our patient population. Therefore, we defined anaemia in both studies as haemoglobin falling $>2$ SD below the mean of age-specific, altitude-adjusted haemoglobin values. .,17 $^{2}$

Food security. Food security is defined as 'all people at all times have both physical and economic access to sufficient food to meet their dietary needs for a productive and healthy life' ${ }^{18}$ We used the HFIAS to assess each child's household food security. Key informants were consulted to ensure that the questionnaire was culturally appropriate and understandable. The survey was adapted by piloting it with mother-child pairs visiting the clinic for one day. The questionnaire was refined in response to their feedback. ${ }^{15,19,20}$ Households were coded into four categories: (i) food secure, (ii) mildly food insecure, (iii) moderately food insecure, or (iv) severely food insecure. We categorised households as 'severely food insecure' (HFIAS=4) or 'not severely food insecure' (HFIAS=1, 2 or 3). ${ }^{21}$ 
Growth and development. Anthropometric data were obtained from patient growth cards, when available, and plotted on World Health Organization (WHO) growth curves. $\mathrm{Z}$ scores were calculated by comparing weight-for-age, lengthfor-age, and weight-for-height. Patients $>2$ SD below the mean were classified as underweight, stunted, and wasted, respectively. ${ }^{22}$

\section{Ethical approval}

Ethical clearance was granted by the ethical review boards of each participating insitution.

\section{Results}

\section{7 results}

Ninety-two children were enrolled; 4 were withdrawn before data could be collected, 1 moved to Johannesburg, and 3 were withdrawn at the request of their fathers. Of the 88 patients remaining, 21 were lost to follow-up, leaving 67 who were screened for anaemia and assessed for food insecurity. Fifteen patients were excluded retrospectively for being clinically ill at the time of sample collection. In sum, 52 well children $<5$ years old were included in the study.

Thirty-two (61.5\%) of the children were boys, and 39 (75\%) were breastfed at the time of enrolment. Median household size was $5 \pm 3.6$ inhabitants. The number of siblings ranged from 0 to 3.3 with a median of 1 . Patients travelled $6 \pm 7.4 \mathrm{~km}$ to reach the clinic.

Thirty-nine (75\%) out of the 52 children were found to be anaemic. The median haemoglobin concentration was $9.65 \pm 2.6 \mathrm{~g} / \mathrm{dl}$. A number of variables were assessed for significance among anaemic children (Table I). Girls were significantly more likely to be anaemic than boys $(20 / 20 \mathrm{v}$. $19 / 32$ respectively; $p=0.001$ ). Height data were available for 41 patients, and weight data for 51 patients. Anaemic children were significantly less likely to be underweight compared with their peers ( $32 / 38$ v. $5 / 12$ respectively; $p=0.007)$. No significant association was observed regarding the remaining variables.

\section{8 results}

Seventy-five mother-child pairs were enrolled; 4 pairs were subsequently excluded, 1 because the child's gender was omitted and 3 because they were $>60$ months old. We analysed only prevalence rates of anaemia among children; nutritional information and the role of anaemia in mothers await final analysis.

Fifty-four $(76 \%)$ out of the 71 children were found to be anaemic. There was no significant difference in anaemia prevalence between THC and PC (13/18 v. 41/53; $p=0.75)$. The gender differences observed in 2007 were not observed in 2008.

\section{Discussion}

Childhood anaemia is treatable and preventable. Unfortunately, the resource-limited regions of the world bear the greatest burden of anaemia. Their paucity of resources to address anaemia necessitates robust surveillance programmes to identify at-risk populations and to monitor interventions to ensure effective utilisation of resources. ${ }^{23}$
We evaluated the prevalence of anaemia among clinically well children $<5$ years old visiting THC in Thohoyandou, Limpopo Province; to our knowledge, this is the first survey of its kind conducted in the region. Three-quarters of the participating children were anaemic. Our preliminary results from a follow-up study of the prevalence of anaemia among mother-child pairs in the same region a year later show a similar prevalence of anaemia (75\% v. $76 \%$ ) among children in the two studies. Intestinal helminths, H. pylori, food insecurity, concomitant illness and cited causes of anaemia did not significantly contribute to the rate observed in 2007. Anaemia was significantly more prevalent among girls in 2007.

SAVACG completed the largest study of anaemia in South Africa in 1994, observing anaemia in 20\% of the 11430 children $<6$ years old. ${ }^{9}$ While our prevalence finding and theirs differ strikingly, the communities they sampled varied greatly in geographical location and socio-economic status, and are therefore not a good comparison.

Agreement between our two data sets validates our argument that anaemia is highly prevalent among our study population. Further comparisons can be drawn from other studies. Faber et al. observed a $65.2 \%$ prevalence of anaemia among children $<2$ years old in rural KwaZulu-Natal. ${ }^{10}$ Simultaneous dietary analysis pointed to diets high in carbohydrates but lacking animal protein, fruit and vegetables. Peri-urban settlements studied near Bloemfontein found 44 $47 \%$ of children to be anaemic, also citing low iron intake as a probable aetiology. ${ }^{13}$ Nutrition is therefore a commonly cited cause of anaemia in children.

The HFIAS was used to assess access to food among households of our patients, $37 \%$ of whom came from severely food-insecure households. However, this factor alone did not seem to contribute to the development of anaemia. We did not evaluate dietary intake in 2007, and results are still pending of the dietary intake evaluated in 2008.

The effect of illness on anaemia is well documented, and we controlled for this by excluding children who were clinically ill at the time of enrolment. We investigated the effects of specific infections on the development of anaemia. Although intestinal helminths often cause anaemia in tropical areas, they did not appear to play a role in 2007. Only one child was found to have hookworms at the time of stool analysis. This low prevalence was observed previously in the area (Stanley: personal communication), and could be a result of both studies having taken place in the dry season. Children who had been dewormed in the previous 6 months were at no less risk for becoming anaemic than those without previous treatment. Intestinal helminths do not appear to play a role in the prevalence of anaemia during the dry season.

Malaria causes anaemia in Africa and is endemic in Limpopo Province, where transmission rates are lowest during June, July and August, and lowest in patients aged $0-4$ years. ${ }^{24}$ Given the timing and target demographics of our study, we do not feel that malaria played a significant role in our results.

A $56 \%$ prevalence of $H$. pylori was demonstrated among children $<5$ years old in Thohoyandou. ${ }^{25}$ As a link has been shown between anaemia and $H$. pylori infection among young children, we tested for $H$. pylori by PCR analysis of stool 
samples. Our prevalence disagreed with previous findings, probably owing to differences in our patient populations; other studies were of children with acute diarrhoeal illness, while ours were well children. H. pylori is more likely to be found in the stools of the acutely ill and malnourished than in healthy individuals, which could account for these differences. ${ }^{26}$

Our most striking finding was the significant difference in prevalence of anaemia between girls and boys in 2007. This result was not replicated in 2008. This inconsistency may be explained by the fact that most patients in the 2008 study were from Pfanani. While the girls from THC were more likely to be anaemic than boys from THC in both years, the girls at Pfanani were not more likely than boys to be anaemic.

There is little literature on the relationship between gender and anaemia in South Africa. Nutritional profiles among Kenyan children also demonstrated higher rates of anaemia among girls, attributing the differences observed to social feeding practices. ${ }^{27}$ While social norms could result in differential intake of iron-rich foods between genders, we did not assess this aspect. Subsequent studies should include detailed dietary analysis and engage experts in sociology and anthropology to better understand this complex issue.

This study has several limitations. The lack of red blood cell (RBC) indices prevents further classification of anaemia, which could have helped in finding an aetiology. The original study did not include this because of uncertainty regarding laboratory facilities, cost, and a lack of understanding of the region's anaemia burden. Another concern was the quantity of blood that was required for a complete blood count (CBC), and its implications for already anaemic children. Our sample size was also small; however, the reproducibility of our findings minimises this as a limitation. We also did not control for HIV infection. Although $20 \%$ of women in Limpopo are HIV positive, prevention of mother-to-child transmission (PMTCT) is covered in approximately $60 \%$ of cases. Therefore, it was felt that the likelihood of seropositive children enrolling was low. Future studies should plan to overcome these logistical and clinical obstacles.

While the ultimate cause is not apparent, the most probable infectious causes (hookworm and malaria) are unlikely. $H$. pylori, another possible cause, was also not associated with anaemia. Therefore, the paucity of iron-rich foods in the diets of children in the area may play a role in the high prevalence of anaemia. Future studies should evaluate the relationship of anaemia and gender, and aim to profile the dietary intake of children in the region.

This study would not have been possible without the support of Matron Vho Angie Mutondwa of THC. We thank Nurses Mathebula and Rambani for lessons on the people, history and health of Thohoyandou, and for their vital role in patient enrolment and sample collection. We thank Vhonani Netshandama, of the University of Venda, for her logistical support and advice. This study was a product of collaboration between University of Venda and University of Virginia Center for Global Health, a relationship facilitated by Professor Robert Swap and the South African Virginia Networks and Associations (SAVANA). Both institutions provided us with wisdom, support and guidance, and we are deeply grateful.

\section{References}

1. Irwin JJ, Kirchner JT. Anemia in children. Am Fam Physician 2001; 64(8): 1379-1386.

2. Sullivan KM, Mei Z, Grummer-Strawn L, Parvanta I. Haemoglobin adjustments to define anaemia. Trop Med Int Health 2008; 13(10): 1267-1271.

3. Walker SP, Wachs TD, Gardner JM, et al. Child development: risk factors for adverse outcomes in developing countries. Lancet 2007 Jan 13; 369(9556): 145-157.

4. Lozoff B, Kaciroti N, Walter T. Iron deficiency in infancy: applying a physiologic framework for prediction. Am J Clin Nutr 2006; 84(6): 1412-1421.

5. Lozoff B, Jimenez E, Hagen J, Mollen E, Wolf AW. Poorer behavioral and developmental outcome more than 10 years after treatment for iron deficiency in infancy. Pediatrics 2000 105(4): E51.

6. Sherriff A, Emond A, Bell JC, Golding J, ALSPAC Study Team. Should infants be screened for anaemia? A prospective study investigating the relation between haemoglobin at 8,12 , and 18 months and development at 18 months. Arch Dis Child 2001; 84(6): 480-485.

7. Levy A, Fraser D, Rosen SD, et al. Anemia as a risk factor for infectious diseases in infants and toddlers: results from a prospective study. Eur J Epidemiol 2005; 20(3): 277-284.

8. Phiri KS, Calis JC, Faragher B, et al. Long-term outcome of severe anaemia in Malawian children. PLoS ONE 2008; 3(8): e2903.

9. South African Vitamin A Consultative Group (SAVACG). Anthropometric, vitamin A, iron and immunisation coverage status in children aged 6-71 months in South Africa, 1994. S Afr Med J 1996; 86(4): 354-357.

10. Faber M, Benade AJ. Nutritional status and dietary practices of 4-24-month-old children from a rural South African community. Public Health Nutr 1999; 2(2): 179-185.

11. Faber M, Swanevelder S, Benade AJ. Is there an association between the nutritional status of the mother and that of her 2-year-old to 5-year-old child? Int J Food Sci Nutr 2005; 56(4): 237-244.

12. Eley BS, Sive AA, Shuttleworth M, Hussey GD. A prospective, cross-sectional study of anaemia and peripheral iron status in antiretroviral naive, HIV-1 infected children in Cape Town, South Africa. BMC Infect Dis 2002; 2: 3 .

13. Dannhauser A, Bester C, Joubert G, et al. Nutritional status of preschool children in informal settlement areas near Bloemfontein, South Africa. Public Health Nutr 2000; 3(3): 303-312.

14. Windle HJ, Kelleher D, Crabtree JE. Childhood Helicobacter pylori infection and growth impairment in developing countries: a vicious cycle? Pediatrics 2007; 119(3): e754-759.

15. Coates J, Swindale A, Bilinsky P. Household Food Insecurity Access Scale (HFIAS) for Measurement of Household Food Access: Indicator Guide (v.2). Washington, USA: Food and Nutrition Technical Assistance Project, Academy for Educational Development, July 2006.

16. Gramley WA, Asghar A, Frierson HF, Jr, Powell SM. Detection of Helicobacter pylori DNA in fecal samples from infected individuals. J Clin Microbiol 1999; 37(7): 2236-2240.

17. Aquino J. Hematology: General evaluation of anemia. In: Custer JW, Rau RE, eds. The Harriet Lane Handbook: A Manual for Pediatric House Officers. 18th ed. Philadelphia, USA: Elsevier Mosby, 2008: 359.

18. USAID Policy Determination: Definition of Food Security. Washington, USA: USAID, 1992. http://www.usaid.gov/policy/ads/200/pd19.pdf (accessed 6 September 2009).

19. Frongillo EA, Nanama S. Development and validation of an experience-based measure of household food insecurity within and across seasons in northern Burkina Faso. J Nutr 2006; 136(5): 1409S-1419S.

20. Swindale A, Bilinsky P. Development of a universally applicable household food insecurity measurement tool: process, current status, and outstanding issues. J Nutr 2006; 136(5): 1449S$1452 S$.

21. Weiser SD, Frongillo EA, Ragland K, Hogg RS, Riley ED, Bangsberg DR. Food insecurity is associated with incomplete HIV RNA suppression among homeless and marginally housed HIV-infected individuals in San Francisco. J Gen Intern Med 2009; 24(1): 14-20.

22. The World Health Organization. Child Growth Standards. Geneva: World Health Organization, 2009. http://www.who.int/childgrowth/standards/en/ (accessed 6 September 2009).

23. Stoltzfus RJ. Rethinking anaemia surveillance. Lancet 1997; 349(9067): 1764-1766.

24. Gerritsen AA, Kruger P, van der Loeff MF, Grobusch MP. Malaria incidence in Limpopo Province, South Africa, 1998-2007. Malar J 2008; 7: 162

25. Samie A, Obi CL, Barrett LJ, Powell SM, Guerrant RL. Prevalence of Campylobacter species, Helicobacter pylori and Arcobacter species in stool samples from the Venda region, Limpopo, South Africa: studies using molecular diagnostic methods. J Infect 2007; 54(6): 558-566.

26. Kabir S. Detection of Helicobacter pylori in faeces by culture, PCR and enzyme immunoassay. $J$ Med Microbiol 2001; 50(12): 1021-1029.

27. Shell-Duncan B, McDade T. Cultural and environmental barriers to adequate iron intake among northern Kenyan schoolchildren. Food Nutr Bull 2005; 26(1): 39-48. 